\title{
Minireview
}

\section{The complex genetic basis of simple behavior}

\author{
Anna J Jasinska and Nelson B Freimer
}

Address: Center for Neurobehavioral Genetics, University of California, Los Angeles, CA 90095, USA.

Correspondence: Nelson B Freimer. Email: nfreimer@mednet.ucla.edu

\begin{abstract}
Genetic approaches to dissecting complex traits in animal models increasingly use transcript levels as a molecular phenotype and as validation for predictions of gene function. A recent study in BMC Biology using these approaches shows the complexity of the genetic contribution to aggressive behavior in Drosophila.
\end{abstract}

\section{Genetic investigation of quantitative behavioral traits}

Neuropsychiatric geneticists are now focusing considerable attention on the investigation of quantitative human behavioral traits, postulating that such phenotypes could be more straightforward to genetically map than neuropsychiatric disorders. Such syndromes are among the most complex of human traits, a fact that may explain why efforts to elucidate their etiology have been singularly unsuccessful. Although quantitative phenotypes may be simpler than clinically heterogeneous diseases, there is still little evidence that human behavioral quantitative trait loci (QTL) will be easy to identify. For this reason there is a tremendous appeal in using the powerful tools available for genetic investigation of simple model organisms; genes implicated in behavioral variation in flies or worms can then be targeted for various forms of analysis in mammals, including humans. This strategy depends on the identification of suitable measures of behavior in simple systems. Circadian rhythms are a classic example. Investigations of clock mutants in Drosophila led to the discovery of the first circadian rhythm gene, period, and ultimately to cellular pathways underlying circadian behavior [1]; this line of investigation was subsequently successfully extended in rodents as well as humans [2].

Aggressive behavior is another complex behavioral trait that can be efficiently modeled in Drosophila [3,4]. Yet, as recently reported by Edwards et al. [5], aggressive behavior in flies results from the action of numerous genes, reflects extensive pleiotropy, and is significantly influenced by molecular processes outside the nervous system. Perhaps circadian rhythm phenotypes will prove more an exception than the rule; when it comes to behavior, a simple system does not guarantee simple genetics.

\section{Utility of fly models of complex behavior}

Given the observation that aggressive behavior in flies has such an apparently complex genetic basis, it is worth reviewing the motivations for using such a behavioral genetic model. In humans it is a given that most behavioral traits involve interactions between numerous genetic loci in the context of multiple, mostly unquantified environmental influences. No strategies implemented so far have been sufficiently powerful to overcome the challenge represented by this degree of complexity, and the systematic genome-wide mapping of human behavioral QTL is, therefore, still a nascent endeavor. This fact alone could account for interest in investigation of behavioral phenotypes in virtually all widely used animal genetic models, including Drosophila. Given the functional analogies of tissues, organs and organ systems as well as the considerable conservation of neurobehavioral traits and of a high proportion of gene counterparts between human and fly [6], identification of Drosophila behavioral QTL is useful for informing studies in other organisms, including humans [7]. Elucidation of the molecular basis of a specific trait such as aggressive behaviour in Drosophila could answer several questions of generalized importance: which genes of unknown function, gene functions and pathways may contribute to the trait? How many genes are implicated in a single complex behavior? What is their effect on the trait in terms of magnitude and specificity?

The particular strength of the Drosophila model for behavioral investigation is its suitability for various experimental genetic approaches. It is ideal for artificial selection for a target trait (such as aggressive behavior) and for genetic modification of candidate genes. In addition, it offers diverse complex behavioral and neuroanatomical phenotypes that can be efficiently quantified to study the putative pleiotropy of candidate genes. For example, a quantitative aggression phenotype has been assayed by scoring aggressive encounters among male files that were exposed to a food droplet after 90 minutes of food deprivation [4,5]. In Drosophila it is also particularly straightforward to control variations in environmental influences that might influence behaviors, a factor that adds substantially to the power to identify genes that influence quantitative traits, especially genes contributing a small amount of the trait variance. 


\section{Genes implicated in aggressive behavior}

As Drosophila has a short generation time and controlled mating is easy, it is possible to perform artificial selection by repeatedly selecting individuals that show extreme scores for behavioral measures. Resulting divergent lines with high or low aggressive behavior show significant differences in gene expression for as much as $10 \%$ of the fly genome [4]. It is likely that genetic variants directly regulate only a relatively small fraction of the genes contributing to the transcriptional response to selection for aggressive behavior; rather, most of these genes are co-regulated in response to causal genes [8]. Nevertheless, the observation that a substantial fraction of the Drosophila genome may be related to aggressive behavior raises interest in determining whether specific genes are directly involved in the trait, in quantifying the magnitude of their effect, and in evaluating whether these genes also contribute to other traits.

The effect of a candidate gene on a complex trait in an animal model can be directly established by functional analysis - for example, by inducing mutation in a candidate gene and then quantifying relevant phenotypes. Unlike QTL mapping, which establishes relations between a phenotype and specific underlying causative genetic variants, mutational analysis discovers the function of candidate genes and elucidates the molecular mechanisms for such functions. The advantage of mutational analysis is that it tests a role of a candidate gene on a defined isogenic background, facilitating the detection of subtle mutational effects. For mutational analysis Edwards et al. [5] used $P$-element insertional mutant fly lines to investigate the effects of a large series of novel candidate genes that had not been previously implicated in aggressive behavior. These genes were selected solely on the basis of gene expression measures obtained after artificial selection for this trait [4] or their known involvement in other complex behavioral traits. Functional evidence supported a role in aggressive behavior of a large fraction (almost 40\%) of these novel candidate genes, but mutations in them exert only small or moderate effects on aggression level [5].

This observation supports the suggestion that the genetic architecture of complex behavior may be remarkably similar in organisms as neurobiologically simple as Drosophila and as complex as humans [9]. There is now a consensus that human neurobehavioral diseases mainly reflect the effects of large numbers of genetic variants of relatively small effect and, therefore, that genetic dissection of such traits will require investigation of samples of considerable size. The findings of Edwards et al. [5] imply that a similar expectation may hold for QTL mapping of aggressive behaviors in humans. Identifying the functional importance of so many variants will obviously be more straightforward in flies than in humans, and progress in dissecting human traits may depend on the degree to which they reflect molecular mechanisms similar to those in their Drosophila counterparts.

\section{Organismal phenotypes and pleiotropic action of behavioral genes}

One of the most interesting findings to emerge from the investigation of aggression in Drosophila concerns the size and localization of mutation-induced expression changes. Edwards et al. [5] found that relatively small transcriptlevel alterations (twofold or less) noticeably affect behavior. Their observation suggests that, in considering intermediate phenotypes for behavioral traits, it would be unwise to ignore transcripts showing a low magnitude of expression alterations. Their results also remind us that an arbitrary division between the brain and the remainder of the body may be unhelpful in efforts to understand complex behavior. Investigations of the spatial expression pattern of wild-type and mutant genes, using decapitated flies, indicated that significant differences between wildtype and mutated genes occurred for all tested genes; however, for the majority of such genes significant differences were detected in the bodies but were not observed in the heads [5]. Accordingly, investigations of human behavioral traits should probably pay greater attention than is typically the case at present to gene expression patterns in peripheral tissues and more vigorously evaluate other types of molecular phenotypes (such as endocrine markers) as intermediate phenotypes. Indeed, the observations in the fly are consistent with, for example, the confirmed role of hormonal regulation in the development and function of the nervous system as well as in levels and types of aggressive behavior in various species, including non-human primates and humans.

A single gene may generate pleiotropy by contributing to a variety of traits. There is existing - although not abundant evidence that a single gene or even a single genetic variant related to neuropsychiatric diseases may contribute to diverse cognitive and neuroanatomical phenotypes. For example, $P E R 3$ is implicated in a rare human circadian rhythm disorder called delayed sleep phase syndrome, but it is also associated with several other circadian phenotypes and with cognitive measures, such as performance tasks assessed during sleep deprivation and brain responses to a working memory task assessed using functional magnetic resonance imaging [2]. A striking observation reported by Edwards et al. [5] is that, in flies, a very large fraction of mutations introduced in genes related to aggressive behavior produce extremely pleiotropic effects, affecting various complex traits. Among the most interesting of these traits are quantitative changes in brain morphology observed in mutants' mushroom bodies. These structures were previously implicated in aggressive behavior in Drosophila and show many parallels to an analogous mammalian brain structure, the hippocampus, which also has an important role in behavior [10]. The observation of 
pleiotropy of particular genes with respect to related traits (such as aggressive behavior and neuroanatomical phenotypes) may provide an important form of corroboration of the role of a given gene in contributing to complex behavioral traits.

Demonstrations of the importance of pleiotropy in the genetic regulation of behavioral variation also provide support for approaches using human genetic mapping that attempt to take advantage of pleiotropy by identifying various intermediate phenotypes (endophenotypes) that link genes and disease. An endophenotype-centered approach has two major potential advantages over genetic mapping of disease phenotypes themselves. First, it is hypothesized that endophenotypes are more directly associated with genetic variation than is disease. Second, unlike disease, endophenotypes can be investigated as quantitative traits and are, therefore, more readily studied in animal models.

The findings of Edwards et al. [5] indicating novel candidate genes and cellular processes for aggressive behavior in the fly model provide useful information about the biology of behavioral traits and also suggest new loci and pathways for studies in other organisms, including humans. The increasing sense that a universal feature of complex behavior may be the contribution of numerous genetic variants of small effect may influence experimental design in human genetic studies of behavioral traits. Furthermore, although it is often assumed that the genetic complexity of the behavioral traits mostly reflects the complexity of the central nervous system, Edwards et al. [5] demonstrated the importance of also considering genes that act mainly in the periphery. Finally, the extensive pleiotropy of behaviorally important genes observed in the fly model may suggest that their human orthologs exert similarly widespread phenotypic effects. These conclusions are not comforting for those attempting to dissect human behaviors genetically, but they offer a reminder that sustained investigation of simple model systems is critically important to these efforts.

\section{Acknowledgements}

Financial support was provided by NIH grants R01RR016300, RL1MH083268, R01 MH075007 and R01NS037484.

\section{References}

1. Konopka RJ, Benzer S: Clock mutants of Drosophila melanogaster. Proc Natl Acad Sci USA 1971, 68:2112-2116.

2. Takahashi JS, Hong HK, Ko CH, McDearmon EL: The genetics of mammalian circadian order and disorder: implications for physiology and disease. Nat Rev Genet 2008, 9: 764-775.

3. Dierick HA, Greenspan RJ: Molecular analysis of flies selected for aggressive behavior. Nat Genet 2006, 38:10231031.

4. Edwards AC, Rollmann SM, Morgan TJ, Mackay TF: Quantitative genomics of aggressive behavior in Drosophila melanogaster. PLoS Genet 2006, 2:e154.

5. Edwards AC, Zwarts L, Yamamoto A, Callaerts P, Mackay TF: Mutations in many genes affect aggressive behavior in Drosophila melanogaster. BMC Biol 2009, 7:29

6. Fortini ME, Skupski MP, Boguski MS, Hariharan IK: A survey of human disease gene counterparts in the Drosophila genome. J Cell Biol 2000, 150:F23-F30.

7. Greenspan RJ, Dierick HA: 'Am not I a fly like thee?' From genes in fruit flies to behavior in humans. Hum Mol Genet 2004, 13 Spec No 2:R267-R273.

8. Hoffmann AA, Cacoyianni Z: Selection for territoriality in Drosophila melanogaster: Correlated responses in mating success and other fitness components. Anim Behav 1989, 38:23-34.

9. Flint J, Mackay TF: Genetic architecture of quantitative traits in mice, flies, and humans. Genome Res 2009, 19:723733.

10. Mizunami M, Weibrecht JM, Strausfeld NJ: Mushroom bodies of the cockroach: their participation in place memory. $J$ Comp Neurol 1998, 402:520-537.

Published: 27 August 2009

doi:10.1186/jbiol172

(C) 2009 BioMed Central Ltd 